\title{
Design and Development of an E-Commerce System in a Rapid Organized Way
}

\author{
Ahmed Yunus ${ }^{1}$, Md Masum² \\ Software Engineer, AlgoMatrix, Sylhet; B.Sc. (Engg.), Department of Computer Science and Engineering, Shahjalal University of Science \\ and Technology, Sylhet-3114, Bangladesh
}

Associate Professor, Department of Computer Science and Engineering, Shahjalal University of Science and Technology, Sylhet-3114, Bangladesh

\begin{abstract}
E-Commerce has become a key factor to turn a country into a digitalized one. As the number of e-commerce is growing more and more, e-commerce systems should be developed in a good fashion with proper aesthetic look, strong security, good searching system with overall fast interaction between the system and the user. This paper traverses all the necessities to develop a proper e-commerce system with available and free development tools and technologies.
\end{abstract}

Keywords: E-commerce, Python, Django, Aesthetics, Responsive Design, MySQL, Database, Shortest Path First, Dijkstra, Adjacent Node, Hierarchical Searching, Template, JavaScript, Brute Force, SQL injection, CSRF token, Tokenization.

\section{Introduction}

E-commerce is a process where people buy and sell products and services via electronic networks using any personal computer. E-commerce lets a seller reach a wide range of customers and also lets a buyer choose from multiple items without any hassle. For these reasons e-commerce has been identified as an important factor for switching to digital lifestyle. As the number of e-commerce is increasing day by day, rapid development is a key factor so that any business organization can develop their own e-commerce system easily and efficiently. A proper e-commerce must meet some conditions to ensure its appealing look on the client side, a strong security on the server side and some other important features. E-commerce can be Business to Business (B2B) or Business to Consumer (B2C) or both. Rapid development of an e-commerce system mainly refers to development of a website which can be accessed via Desktop and portable devices at ease. A website mainly consists of a frontend on which the user interacts and also a backend which is mainly the server where all the main logical functionalities are executed. This paper mainly focuses on design and development of a B2C e-commerce system to enforce the local businesses to expand nationally and globally.

\section{Literature review for E-commerce Development}

Before starting any development of a software whether it is a website or an application, literature review which is in this case Background Analysis is mandatory in order to resolve the obstacles. An e-commerce system does not differ from that. A background analysis of an e-commerce system includes the stakeholders, their requirements, feasibility study of rapid development of the e-commerce system and most importantly the overall interaction between the customers and the system.

\subsection{Stakeholders}

There are at least 3 stakeholders in a B2C e-commerce system. They are mainly:

\section{Administrators}

Managing the system is the main task of this stakeholder. This means that the administrators are responsible for overall server maintenance and also manage databases, edit databases and add new administrators.

\section{Customer}

This kind of stakeholder is the one who pays something to consume goods and services produced or delivered through the system. The customers will search for their desired product and the system will give its best effort to find the product according to the customer's choice.

\section{Employee}

This stakeholder actually has two roles. One is a managerial post and another one is a delivery man post. The manager does the interaction with the customers and employees in order to deliver products from the warehouse to the individual's house. They also do all the staff that is related with product adding, editing and more. They also assign delivery men to deliver the products to customer's houses. The delivery man is the one who delivers products to customer's houses when they are assigned.

\subsection{Feasibility Study}

The general concept of feasibility is associated with projects of all kinds (housing, marketing, ecological, scientific, industrial...), and specifically bound to an engineering approach to project development. Whenever a project of any kind is to be carried out systematically, through careful consideration and -wherever possible - measurement of the parameters involved, a feasibility study becomes an integral part of the project work. An important question to be taken into account is that a feasibility study should be relatively cheap and quick, and it should inform the decision of whether to go ahead with a more detailed analysis. There are 


\section{International Journal of Science and Research (IJSR) \\ ISSN: 2319-7064}

ResearchGate Impact Factor (2018): 0.28 | SJIF (2019): 7.583

two dimensions which makes the rapid development of ecommerce system as a feasible one:

\section{Technology}

A system stands on how well furnished technologies are there. Many programming languages with proper Framework are available to develop a system. These Frameworks are made by thousands of contributors around the world which have strong security implemented. Besides for e-commerce systems there must be proper payment gateway which are also available to use. SSL certificates are available to use to encrypt data. So technically e-commerce systems are completely feasible.

\section{Resources}

Complete and well suited frameworks or CMS, database with secured connection etc. resources are completely available for free and ready to use in this system.

\subsection{Requirement Analysis}

In order to get a full view of interactions between the stakeholders and the system, requirements define what most are needed.

\section{Customer Perspective}

- E-commerce system interfaces must have appealing look

- The system should be self-explanatory

- The system must be ease to use

- The system must be secured for any kind of transaction

- The system must be secured for login and registration system

- The system must be accessed in multi-platform using desktop or portable devices

- The system should response as fast as possible to customer requests

- As this is E-commerce system there must be a cart system also the customers must have the ease of placing the order from cart

- There must be system so that customers can track their orders

- The customers can easily communicate with the authority for any kind of queries

\section{Administrator Perspective}

- Easily handle database

- Handle design of different platforms

- Add new admins

- Overall maintenance of the system

\section{Employee Perspective}

- Managers can add new product easily

- Managers can edit product details easily

- Managers can sort the orders based on some dimensions and assign it to delivery man

- Managers can answer the queries of customers easily

- Delivery man can easily find out their assigned orders

- Delivery man can easily navigate to customer's address to deliver product

\section{Additional Requirements}

- The system should response to customer support as soon as possible

- The system should have customer profile system for every customer

\subsection{System Architecture}

System design mainly includes the overall interaction procedure between the users and the e-commerce system. To properly shed the light two diagrams namely Activity Diagram and Class Diagram are given. Moreover Class Diagram also represents how the Database should be designed in an e-commerce system.

\section{Activity Diagram}

Activity diagram defines user's interactivity with the system. In Figure 1, the interactivity of the customer along with the delivery system is given below.

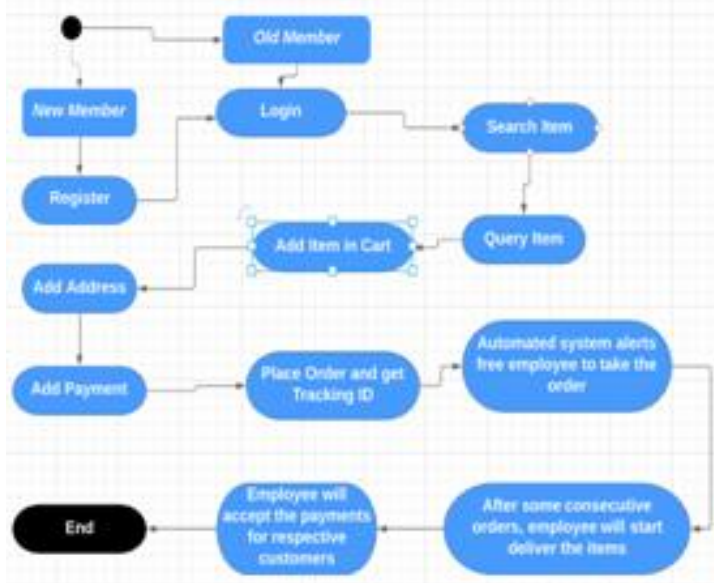

Figure 1: Activity Diagram

\section{Class Diagram}

A class diagram is a type of static structure diagram that describes the structure of a system by showing the system's classes, their attributes, methods and the relationships among objects. A class diagram also represents the database structure of a system.In Figure 2, the class diagram of an ecommerce system is displayed.

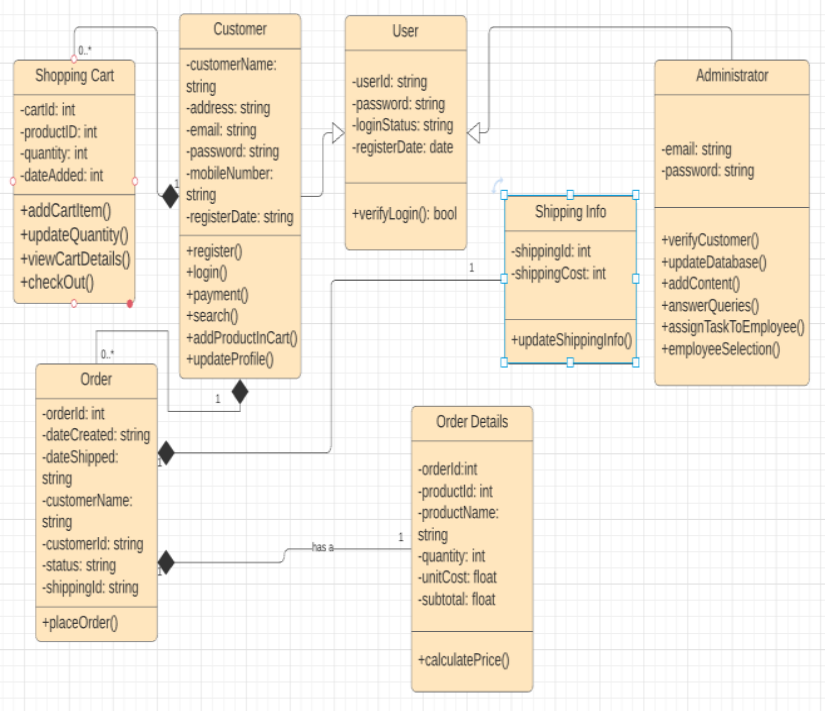

Figure 2: Class Diagram 


\section{International Journal of Science and Research (IJSR) \\ ISSN: 2319-7064}

ResearchGate Impact Factor (2018): 0.28 | SJIF (2019): 7.583

\section{Development Tools}

The main phase of rapid development of an e-commerce system starts with choosing the right development tools among the many. There are CMS (Content Management System), Frameworks and also raw coding for server development. CMS and Frameworks are definitely winners against raw coding for server development as those have a systematic organized way for development of a website. So then comes the choice between Frameworks and CMS.

\subsection{CMS vs Framework}

Below the chart shows the differences between Frameworks and CMS [1]

\begin{tabular}{|c|c|}
\hline CMS & Framework \\
\hline $\begin{array}{c}\text { CMS has a predefined set of } \\
\text { features, to choose from an } \\
\text { already styled theme for a } \\
\text { website and you can add new } \\
\text { features by easily installing } \\
\text { plugins. CMS' typically have } \\
\text { limitations }\end{array}$ & $\begin{array}{c}\text { With a framework, } \\
\text { everything has to be built } \\
\text { from scratch but creation of } \\
\text { distinctive and unique } \\
\text { features is easier than } \\
\text { framework. Frameworks } \\
\text { are highly customizable }\end{array}$ \\
\hline $\begin{array}{c}\text { Since websites developed } \\
\text { using frameworks will } \\
\text { feature their own custom } \\
\text { code that is integrated into } \\
\text { the framework, it's much } \\
\text { harder to find security } \\
\text { means the code is available to } \\
\text { anyone. At first glance, this is a } \\
\text { good thing, but it also makes the } \\
\text { job of hackers and anyone with a } \\
\text { malicious purpose a little easier. } \\
\text { frameworks come with } \\
\text { built-in functions like SQL } \\
\text { injection or Cross Site } \\
\text { Scripting that allow for } \\
\text { encryption and protection } \\
\text { against the most common } \\
\text { types of attacks. }\end{array}$ \\
\hline $\begin{array}{c}\text { CMS' can be adapted to have a } \\
\text { lot of features through the use of } \\
\text { plugins which are basically third } \\
\text { party plugins. }\end{array}$ & $\begin{array}{c}\text { Frameworks can be molded } \\
\text { to any needs and any } \\
\text { requirements. }\end{array}$ \\
\hline
\end{tabular}

CMS' are easier to use if the project requirements are relatively straightforward. In many cases, all that's required is downloading and installing the software, and the system is immediately up and running. But an e-commerce system's requirements are not relatively straight. The requirements stated on chapter 2 clearly indicates that developing an ecommerce system cannot be properly done with CMS. There are customized requirements for which customized codes must be written. So here the first choice would go for a proper framework.

\subsection{The right Framework}

There are also many frameworks from which to choose the suitable one is a somewhat difficult task. Some popular frameworks are Django framework which backend language is Python, Laravel which backend language is PHP and Rails which backend language is Ruby. Some other frameworks are Spring, Angular JS, Flusk and many more. For a rapid development purpose Django is the most suitable one which is stated below:

- Django is a stable framework
- Django uses Python which has great community backup and also a stable language

- As for e-commerce systems, many Machine Learning related tasks are embedded in the system and Python is best for having good and stable Machine Learning libraries.

- Django has a built in Admin board system.

- Django has strong security integrated into the framework like SQL injection prevention, Cross-site scripting prevention, $\mathrm{X}$-Frame prevention etc.

- Django supports different kinds of Database like MySQL, PostgreSQL etc.

- Django uses MVT architecture which is a reflection of popular MVC architecture

\subsection{Architecture of Django framework}

The MVT (Model View Template) is a software design pattern. It is a collection of three important components Model View and Template. The Model helps to handle databases. It is a data access layer which handles the data. The Template is a presentation layer which handles the User Interface part completely. The View is used to execute the business logic and interact with a model to carry data and render a template. Although Django follows MVC pattern but maintains its own conventions. So, control is handled by the framework itself. There is no separate controller and complete application is based on Model View and Template. That's why it is called MVT application. [2]

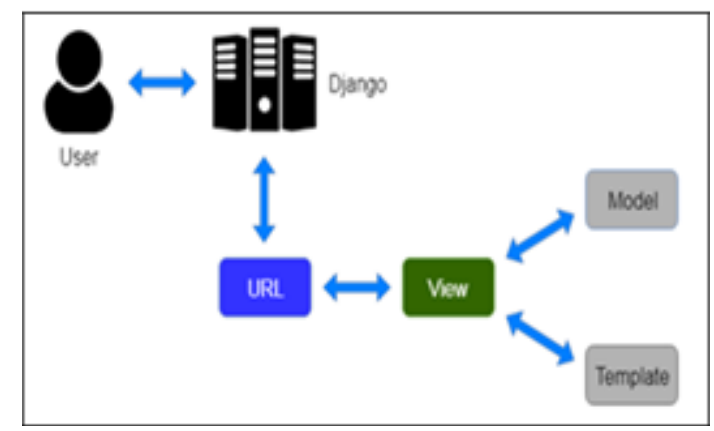

Figure 3: Django MVT control flow

\subsection{Database}

MySQL is a relational database which is best suitable for rapid development. Xampp which is developed by Apache has a nice visual row and column structure in order to understand the whole database. Besides that, Django totally supports MySQL. So for rapid development Xampp software which enables MySQL is chosen for e-commerce systems.

\section{Frontend design}

For an e-commerce system, proper aesthetic visual is a key factor for the success of an e-commerce business. People tend to visit websites again and again if the website is visually empowered. But it is a most important concern that the pages must load fast. It is said that 2 seconds is the threshold for ecommerce website acceptability [3]. So this must be considered while designing the pages. The product images should be resized. There should not be any 


\section{International Journal of Science and Research (IJSR) \\ ISSN: 2319-7064}

ResearchGate Impact Factor (2018): 0.28 | SJIF (2019): 7.583

unnecessary JavaScript function execution which delays the loading time of a web page.

Django Framework supports HTML and CSS for designing the frontend. Bootstrap is the most popular CSS Framework for developing responsive and mobile-first websites which is also supported by Django Framework. Using Bootstrap decreases the leverage of designing. There are figures included below which describes three main pages of an ecommerce website.

\section{Homepage}

Homepage is the first page of a website. It is necessary to show as much information to attract the customers while not losing the loading time. Page must be loaded within 3-4 seconds max with all the images, CSS, HTML and JavaScript code. The top portion should include the search bar, following that some main categories. Then comes the list of products. And lastly a footer. Below two images show how to properly design a homepage.

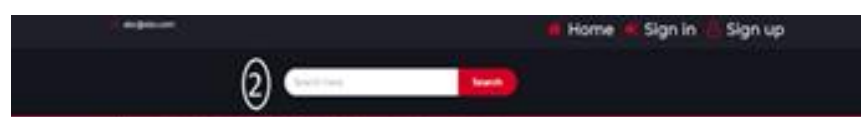

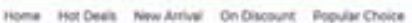

AL CATECORIES

(1)
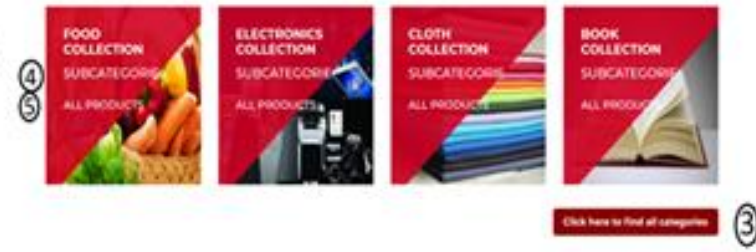

Figure 4: Homepage upper part

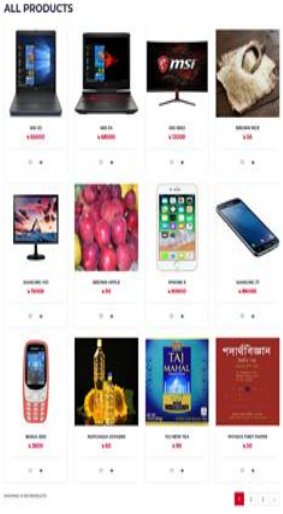

Figure 5: Homepage middle part

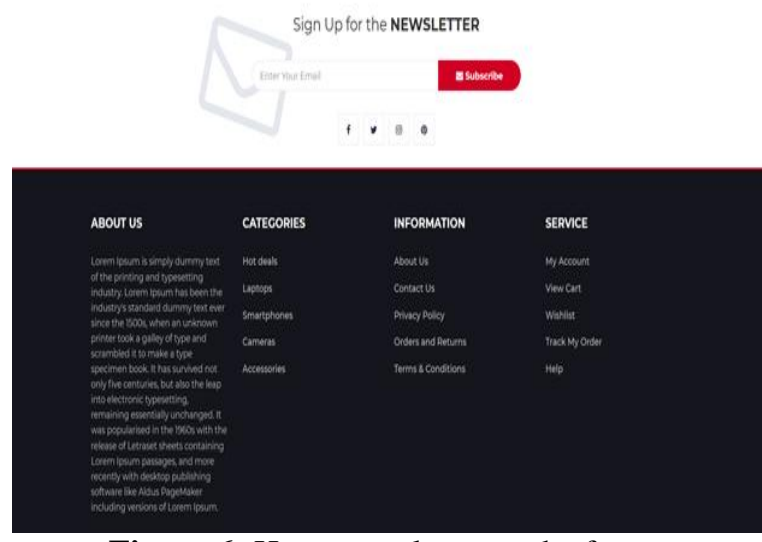

Figure 6: Homepage last part the footer

- Marker 1: defines the total blank space that should be available on both sides of the Homepage. Using Bootstrap makes this space automatically.

- Marker 2: defines that Search Bar should be on the top.

- Marker 3: lets a user find a product categorically. This will be briefly discussed on the Searching section.

- Marker 4: lets a user find the subcategory of that main category.

- Marker 5: lets a user find the products under that main category.

- It is necessary to limit the network usage on the client side. If the images are $600 \times 600$ pixels, and if 30 products are shown at once it would take $420.22 \mathrm{~KB}$ to load the page. This is totally acceptable and also it would take less than threshold time to load the homepage.

\section{Product Page}

Figure 7 defines how a product page should be displayed.

- Marker 1: defines how the products along with extra images should be displayed with proper padding and space.

- Marker 2: shows the Cart and Wishlist options. These are enabled when the user does login with proper credentials. Clicking the 'Add to Cart' button lets a user add a product in cart.

- Marker 3: defines the review box where customers can review a product.

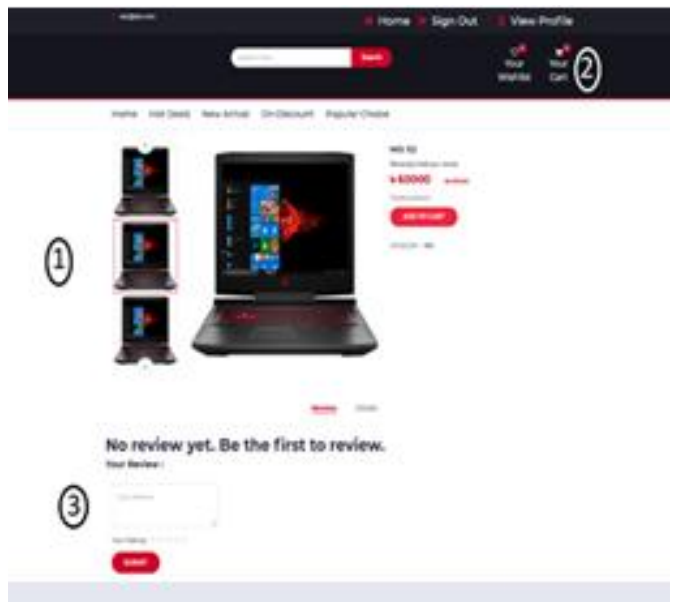

Figure 7: Product Page

Volume 9 Issue 3, March 2020 www.ijsr.net 


\section{International Journal of Science and Research (IJSR) \\ ISSN: 2319-7064}

ResearchGate Impact Factor (2018): 0.28 | SJIF (2019): 7.583

Delivery Tracking Page

Another important page that must be present for the customer's website is delivery tracking. After completing an order, there should be a system where customers can track their order. In Figure 8, there are two columns for each order, those are Delivered and On the way. These are discussed in section 7 Delivery System.

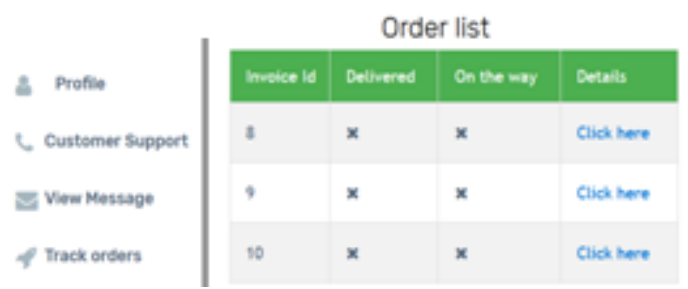

Figure 8: Product delivery tracking page

\section{Searching System}

Success of an e-commerce system heavily relies on a proper searching system. People tend to search for a product directly rather than browsing all the products to find their desired one. A simple but very functional searching system can be made by only 'like' keywords from MySQL. This paper proposes four kinds of searching systems in order to make ease for a customer to find the product.

\subsection{Direct product search}

Direct product search lets a user type the product name in the search box that is shown in Figure 4, marker 2. Then this string will be taken as input in Ajax Query and sent to Django server which will look for string matching with the product name using SQL 'like' keyword. If there is any valid match it will drop down the product name along with images like below in the figure.

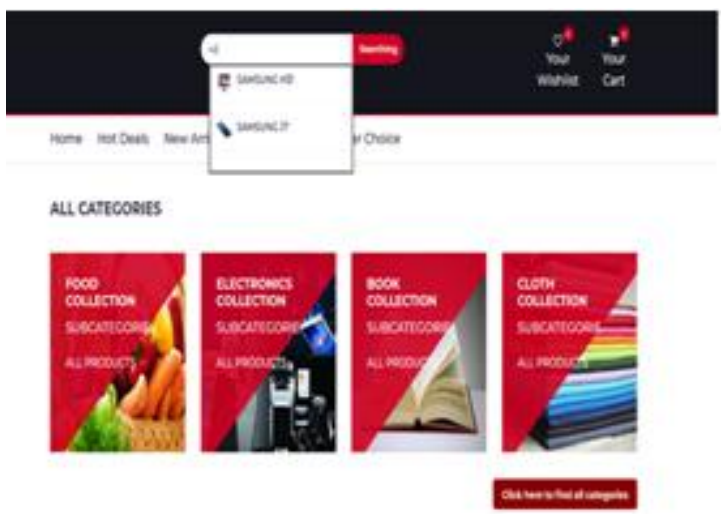

Figure 9: Direct searching drop down system.

\subsection{Direct category search}

Before illustrating on direct category search first Hierarchy Product Insertion method should be described. As this is an e-commerce system, and the website will render different types of products, products must be inserted into the system in a systematic organized way so that the products can be searched easily. Every product has some upper level. And every product can be represented using a hierarchy fashion. For example, Samsung S9 mobile can be presented like this:
Electronics $\rightarrow$ Mobile $\rightarrow$ Samsung $\rightarrow$ Samsung S9. Another example is, Food $\rightarrow$ Grocery $\rightarrow$ Rice $\rightarrow$ Brown Rice. It can be said that any product in this world can be categorized within at most 6 levels, actually not more than 5 levels. 1 level is kept extra in the system if any product crosses the 5 level limit. Below image clarifies how Hierarchical fashion works:

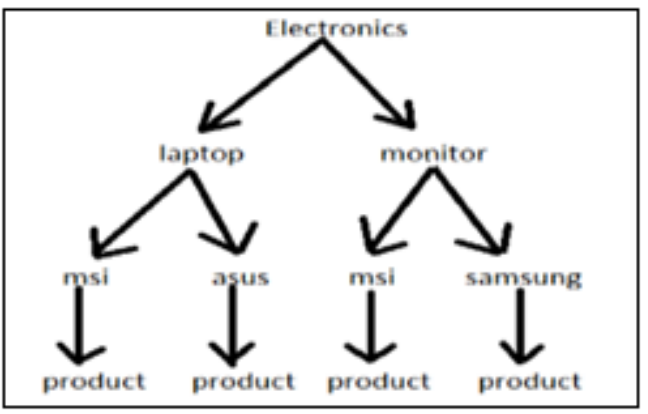

Figure 10: Hierarchical system in product storing

So when a customer searches for example 'msi' keyword, and clicks the search button, it will hit the server and starts searching with this keyword in every level using MySQL 'like' keyword. And if it finds the match with any level it will fetch the whole product list of that level and displays it as a search result.

\subsection{Category to category search}

Figure 10 displays how the hierarchical fashion is used in product insertion and searching. But if the brand name like 'msi' or 'samsung' is being used as a key for finding levels and products then there will create an ambiguity. There are many brands which make different types of products. For example, MSI brand makes both monitors and motherboards. Samsung makes Solid State Drive (SSD) and mobile phones. Now in Figure 4, there are categories that are on the top of the hierarchy. Clicking on subcategory will reveal the subcategory of that category on another page. For example clicking the Electronics will show this page:

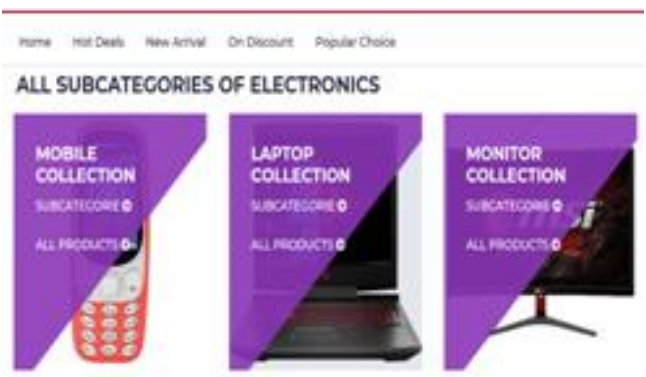

Figure 11: Displaying subcategory

Now clicking on the subcategory of Laptop or Monitor both will show some common brands like MSI. Now if 'MSI' is being clicked, according to section 5.2: Direct category search, it will fetch all the products of MSI whether it is laptop or monitor. But as the user clicked the laptop $\rightarrow$ msi, now it must fetch only the MSI laptops, not MSI monitors. So here 'msi' keyword is creating ambiguity. In order to overcome this, a very simple solution is proposed. When inserting the data into the database, every category and their subcategory must have a unique identification number. If a brand has more than one upper level, for example: MSI has

\section{Volume 9 Issue 3, March 2020}




\section{International Journal of Science and Research (IJSR) \\ ISSN: 2319-7064}

ResearchGate Impact Factor (2018): 0.28 | SJIF (2019): 7.583

both 'Monitor' and 'Laptop' as its upper level hierarchy, MSI must be inserted twice. While inserting the brand name it must have an identification number. So Laptop $\rightarrow$ MSI and Monitor $\rightarrow$ MSI will have completely different identification numbers. The below figure clarifies this:
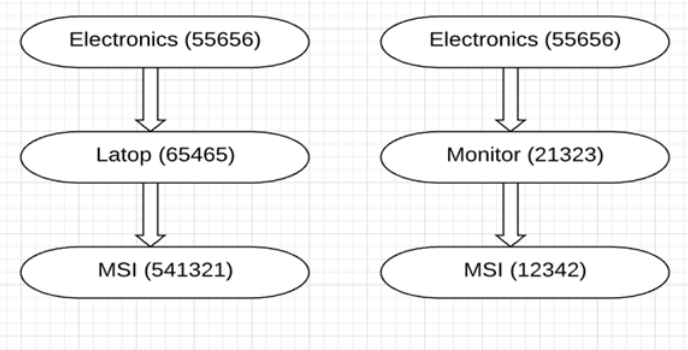

Figure 12: Hierarchical fashion using unique identification number

The root categories that are displayed, have a GET-URL link which sends its unique identification data to the server. The server searches the identification number and searches the product ids which have the same identification number as brand name tag. Finding the list, then it will fetch the product list and send it back to the user.

\section{Strong Security Implementation}

Security is the most essential part of an e-commerce system as there will be many kinds of transactions. Also there must be data safety assured in the system. For these purposes various actions must be taken.

\begin{abstract}
Mobile based login system
After successful registration a verification code will be sent to the customer's mobile number in order to verify that the user is not fake. As there are available sites which provide temporary email addresses, e-commerce systems should introduce mobile and password based login systems rather than email and password based login systems.
\end{abstract}

\section{Separated admin and consumer website}

Websites where Managers add, edit products is the most sensitive part of an e-commerce system because this has access to all kinds of product databases. That is why this server should be separated from normal customer servers and should have restricted access to that server. Only allowed IP's can connect to the admin server which prevents any kind of intrusion in that server.

\section{Brute force prevention}

An intruder can try to access another's account by brute force. To prevent this, if any customer inserts password wrong for a limited time his account will be blocked and will be reopened by inserting verification code sent to his phone.

\section{SQL injection prevention}

Rather than fetching, inserting and updating data in raw SQL, Django ORM queryset must be used in order to stop SQL injection. Django's querysets are protected from SQL injection since their queries are constructed using query parameterization. A query's SQL code is defined separately from the query's parameters.

\section{Clickjacking protection}

Clickjacking is a type of attack where a malicious site wraps another site in a frame. This attack can result in an unsuspecting consumer being tricked into performing unintended actions on the target site. Django contains clickjacking protection in the form of the X-Frame-Options middleware which in a supporting browser can prevent a site from being rendered inside a frame.

\section{Session hijacking prevention}

As this website runs with SSL certificate all the data sent to clients are encrypted. So there is not fear that the session can be hijacked by anyone.

\section{Cross site request forgery protection}

CSRF attacks allow a malicious consumer to execute actions using the credentials of another consumer without that customer's knowledge or consent. Django has built-in protection against most types of CSRF attacks.

\section{XSS prevention}

XSS attacks allow a consumer to inject client side scripts into the browsers of other consumers. This is usually achieved by storing the malicious scripts in the database where it will be retrieved and displayed to other consumers, or by getting consumers to click a link which will cause the attacker's JavaScript to be executed by the consumer's browser. Django ORM queryset and stripping away some special characters to prevent XSS attacks on this server.

\section{Tokenization}

As this is an E-commerce system, it is expected that the search keywords will not exceed more than 4-5 words. So if the search word exceeds more than 5 words it is possible that some kind of attack is happening. So tokenizing the input word and getting length; if the length is 5 or more than the search key word is removed and cannot be executed in the system.

\section{Delivery System}

A complete e-commerce system must have a proper, fast and efficient delivery system to deliver products. The below Figure 13 shows that places can be traversed from base using multiple ways but optimal ways must be selected in order to fast and efficient delivery.

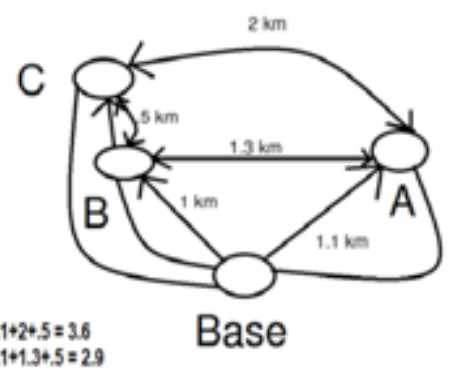

Figure 13: Routing of places

Now-a-days Google maps has been integrated in almost every delivery system. But the problem relies that Google

\section{Volume 9 Issue 3, March 2020}




\section{International Journal of Science and Research (IJSR) \\ ISSN: 2319-7064}

ResearchGate Impact Factor (2018): 0.28 | SJIF (2019): 7.583

maps is not completely free and one has to pay a subscription fee every month. An e-commerce system relies heavily on less expense. A simple clustering using customized Dijkstra algorithm can properly work as a delivery system algorithm.

Dijkstra's algorithm (or Dijkstra's Shortest Path First algorithm, SPF algorithm) is an algorithm for finding the shortest paths between nodes in a graph, which may represent, for example, road networks.

For an e-commerce system, nodes are the places where the products will be delivered. To find the shortest paths among places, distance is a must necessity. This can be achieved manually from Google Maps and this data collection is only for one time but can be reused for any number of iterations. To demonstrate how this delivery system works some real world data has been chosen.

The area that has been chosen is Sylhet District Uttar Surma, Bangladesh which is approximately $14 \mathrm{~km}^{2}$. For mock data, only red circled areas will be chosen. Google recently updated its desktop version with a new feature that lets users measure the distance between two or more points on the map. To toggle this option, right-click on a starting point on the map and select "Measure distance," and then choose a destination to see the distance between the two points. This manual method does not take much time. It may take up to 72 hours to include all the places including narrow areas of a $14 \mathrm{~km}^{2}$ Sylhet area. Only 12 places have been chosen for mock data from the red circle are below in Figure 14. Distances are calculated by the above stated method.

First and foremost place data collection starts from the base where the delivery of products will start. Then from that place, adjacent places are taken and their distance is calculated and saved in a database. In Figure 13: the base's adjacent places are B and $\mathrm{A}$. Then the next step to take adjacent places of those places. Here B's adjacent place is only $\mathrm{C}$ and A's adjacent place is only C. This method is exactly reflected on real world data. For example in Figure 14, Kumarpara has adjacent places of Naiorpul, Eidgah, Charadighirpar, Mirabazar and Saodagortola. These are taken first and then moved to the next step.

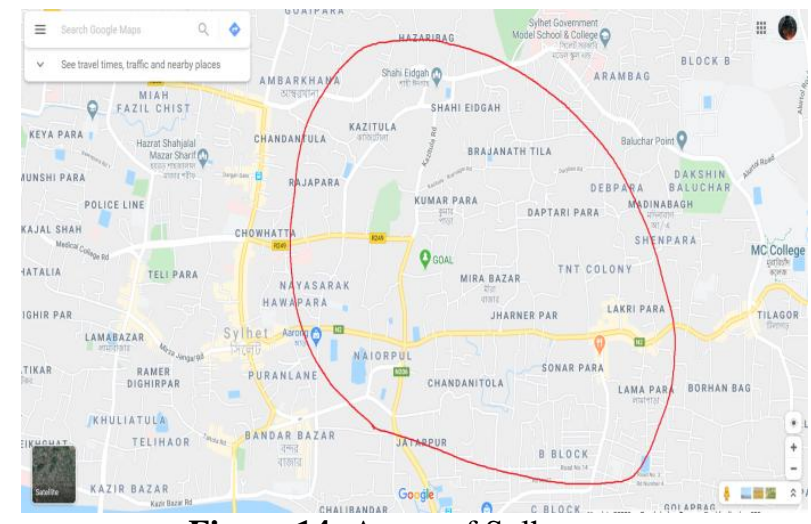

Figure 14: A part of Sylhet area

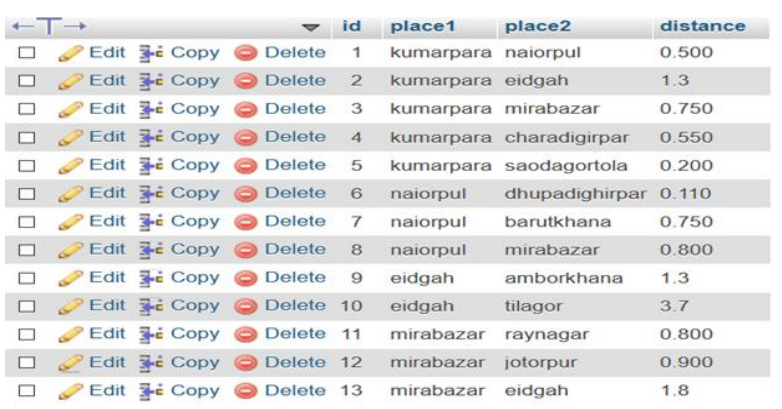

Figure 15: Distance between places.

The mock data has been collected and now it is time to feed them into the Dijkstra algorithm. Dijkstra does not find all the shortest routes between all the nodes. But it looks for the shortest path from one node to another. So the Dijkstra algorithm is slightly customized in order to use it in the system. It takes one place as a base first. And then calculates distances between every place and the base. It re orders them as a list according to distance in descending order. So the first element of the list is the shortest place from base. And then the base is removed as it is traversed. Then the next shortest place becomes the base from where the next place should be chosen. Thus it traverses all the places one by one and finds the shortest routes from one place to another. The algorithm snippet is given below:

initial a list named order_list setorder_list with all pending orders place, ids setbase_station $=$ warehouse location

initial a list named final_result

fori=0 to length(order_list)-1: iforder_list(i) is final_result : pass

else : initial a list named temp_list

for $j=0$ to length(order_list)-1:

compute shortest path using Dijkstra(base_station, order_list $(j))$

store distance results, place name and route in temp_list as tuple

sorttemp_list in descending order storebase_station, temp_list[0] and route in final_result as tuple

setbase_station $=$ temp_list $[0]$

cleartemp_list

returnfinal_result

Three more figures are given below where first one shows how the orders are taken along with the delivery address using drop down menu where drop down menu's list is generated by pre inserted delivery areas, second one shows that Administrators are sorting orders with just one click and third one shows the orders along with their shortest routes.

The database table snippet of distances is given below: 


\section{International Journal of Science and Research (IJSR)}

ISSN: 2319-7064

ResearchGate Impact Factor (2018): 0.28 | SJIF (2019): 7.583

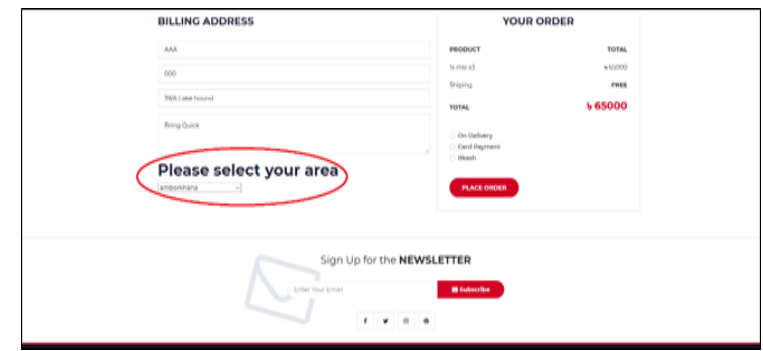

Figure 16: Using drop down menu to select customer's address

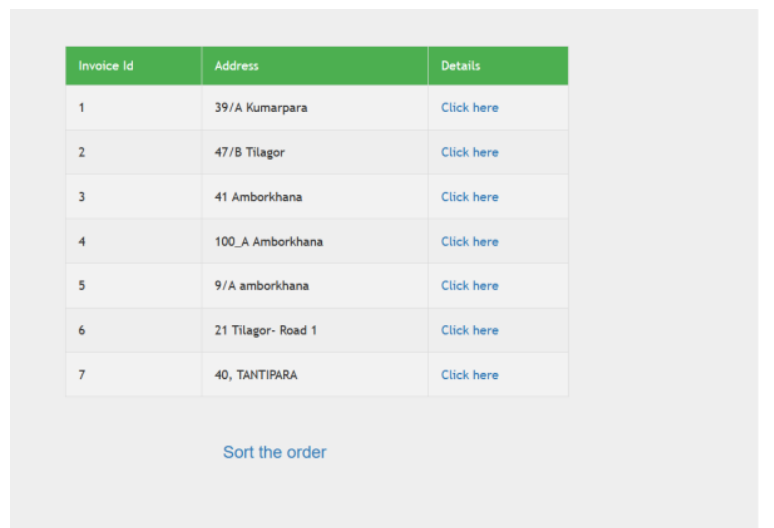

Figure 17: List of unsorted orders from customers.

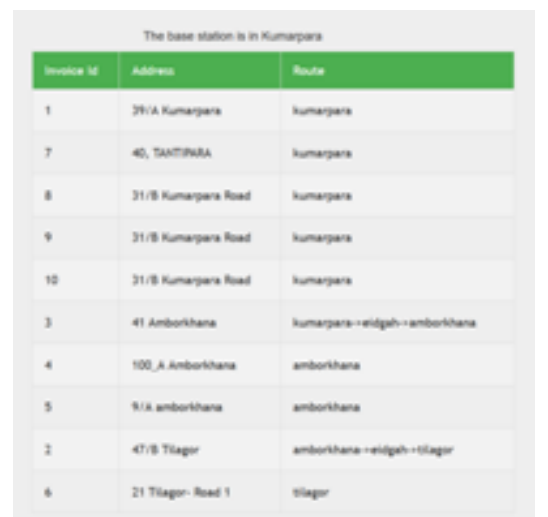

Figure 18: List of sorted orders according to distance in descending order from customers

So the manager has sorted the orders, and these orders have formed clusters. Manager can assign any number of orders like 5 or 6 to individual employees to deliver the products. These 5 or 6 orders will be the closest orders, then next 5 or 6 will be closest and so on. This is how the free routing system works. After the assigned order there will be a mobile app which displays the pending orders with shortest routing. Figure is given below:

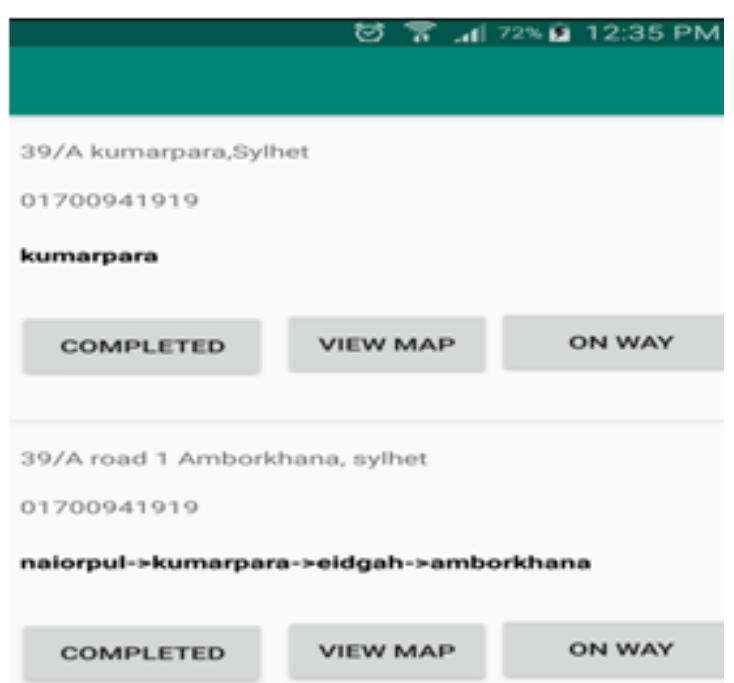

Figure 19: Delivery app which shows pending orders

Clicking 'On the way' button notifies the customer that the order is on the way. After proper delivery, the 'Delivered' button will be pressed by the delivery man.

Clicking the 'View Map' will load Google map which pin points customer's location and delivery man's current location. This is completely free from Google map as routing is not enabled here. Routing is calculated by the Dijkstra method. So this makes a proper, free delivery system.

\section{Some other aspects}

There are some other aspects that must be mentioned:

While adding products in cart, using JavaScript will send the product information and add the product in cart without loading the whole page. JavaScript should also be used in product increment, remove from cart etc.

Homepage should not be overwhelmed with too many products. The homepage should have places to breathe.

\section{Conclusion}

E-commerce has become a necessity in the modern world as all the services are becoming digitalized day by day. But in order to make a successful one, this paper proposes and traverses all the key aspects of an E-commerce system along with design and development methods.

\section{References}

[1] https://www.opensourcecms.com/content-managementsystems-vs-frameworks

[2] https://www.javatpoint.com/django-mvt

[3] MaileOhye, from Google, https://www.bluecorona.com/blog/how-fast-shouldwebsite-be

[4] https://machinelearningmastery.com/prepare-text-datamachine-learning-scikit-learn/

[5] https://en.wikipedia.org/wiki/Dijkstra\%27s_algorithm 